\title{
论规划研究的技巧
}

The Craft of "Doing Research" in Planning

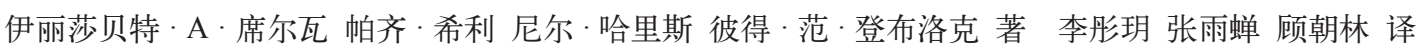
Written by Elisabete A. Silva, Patsy Healey, Neil Harris, Pieter Van den Broeck, Translated by Li Tongyue, Zhang Yuchan, Gu Chaolin

摘要: 这篇文章集中讨论空间和区域规划学科的研究问题。规划研 究与一般的科学研究相同也不同, 甚至与空间研究学科的研究方法 也存在较大差异。文章重点就如下问题进行了论述：(1) 关于规划 学科研究的争论; (2) 空间和区域规划做研究的特殊性; (3) 规划 学科的知识传统及其对研究实践的影响; (4) 规划研究的认识论和 道德敏感性; (5) 在规划实践中研究: 规划研究与规划实践的相互 作用。

Abstract: This paper focuses on "doing research" within the discipline of spatial and regional planning. There are some important differences of research methods in the planning field and other spatial disciplines. The paper addresses on followings: (1) the argument for focusing on research in the planning discipline; (2) the specificities of doing research in spatial and regional planning; (3) intellectual traditions in planning and their impacts on research practices; (4) epistemological and ethical sensibility in planning research; (5) research in practice: the interaction between research and practice.

关键词：规划研究方法; 空间与区域规划; 规划的知识传统

Keywords: Planning Research Methods; Spatial and Regional Planning; Intellectual Traditions in Planning

本文原为劳特利奇 (Routledge) 出版社出版的《规划研究方法手 册》(The Routledge Handbook of Planning Research Methods) 的导言, 译文有所删减。原书中文版由中国建筑工业出版社出版。中国建筑 工业出版社授权我刊发表中译文节选。

作者：伊丽莎贝特· A · 席尔瓦, 英国剑桥大学土地经济系, 高级讲师 帕齐·希利, 英国纽卡斯尔大学建筑、规划和景观学院荣誉退休 教授

尼尔・哈里斯, 英国卡迪夫大学卡迪夫规划和地理学院高级讲师 彼得·范·登布洛克, 比利时鲁汶大学规划与发展研究小组 $(\mathrm{P} \& \mathrm{O} /$ D) 博士后研究员

译者: 李䑣玥, 清华大学建筑学院, 博士研究生 张雨蝉, 曼彻斯特大学建筑学系, 博士研究生 顾朝林, 清华大学建筑学院, 教授

\section{1 关于规划学科研究的争论}

研究分为很多不同形式, 并在多样化的环境中进行。你可 能是规划学院或城市与区域规划系的博士生, 可能是正在撰写 研究生或者本科生论文的学生, 可能是刚刚从业的规划研究人 员, 也可能是才进人规划学科领域从事科学研究的新手, 也许 正想知道如何在规划学科中应用你的技巧和经验, 如何得出规 划学科的专门特征和研究传统。你可能在研究所从事实际的应 用研究工作，也可能已经确定或者被指定就某一 “主题” 进行 研究, 抑或你对怎样研究已经有了初步的想法。非常可能的是 你已经完成或正在进行有关“研究设计”课程或课程模块的学 习, 乃至已经完成或正在做你的研究中的 “研究方法” 部分, 已经涉及大量的社会科学研究方法的文献（Outhwaite \& Turner, 2007; Lewis-Beck et al., 2004; Somekh \& Lewin, 2011; Ruane, 2004)。

这种研究方法的文献的确有用, 但往往比较笼统。教科 书中有些材料可能是为了专门的学科进行专门的研究设计或研 究方法进行描述的, 这对我们规划学科来说往往觉得有点遥 远。阅读这些文献你会发现这些特定的研究设计和研究方法仅 适用于特定的领域, 包括刑事司法、心理学、护理学及社会 工作 (Logio et al., 2008; Lyons \& Coyle, 2008; Clamp et al., 2004; Thyer, 2010)。然而, 有关规划领域研究方法的讨论还没有, 最 接近的其他空间学科的研究方法可能是人文地理学的研究方法 (Flowerdew \& Martin, 2005), 两者有一些共同的特征, 但仍 然差异巨大。我们认为这些差异非常重要, 如果忽视规划学科 做研究的特殊性, 可能会引发一些严重的后果。这些后果包括 学生和研究者在探索和运用全面的研究方法来理解广泛的规划 问题时, 可能会忽视学科本身和在发现世界的过程中采用的研 究方法之间的相互作用。虽然在一些文献中, 规划研究者描述 出他们已有的工作方法 (Roy, 2003; Healey, 2007), 但是仍然缺 
乏规划领域研究的针对性。因此, 毫无疑问, 本书主要聚焦 于规划领域承担研究需要面临的多重挑战, 填补了规划学科 的研究方法这一空白。读者在阅读本书的同时也应该阅读一 些有丰富价值的相邻社会科学研究文献, 尤其一些规划学者 如费吕博基格（Bent Flyvbjerg）在 2001 和 2012 年的研究成 果, 他们在这些方面已经取得了显著的学术贡献。本书被设 计成将广泛的研究设计和研究方法文献与规划领域的特殊性 关联起来的规划研究方法实用手册。在这本书中我们试图提 供一些特别的章节, 要求作者特别注重规划领域做研究工作 的技巧、应用在规划领域的各种各样研究方法以及规划中做 研究产生的特定挑战。

\section{2 空间和区域规划做研究的特殊性}

已知有关研究的最常用的定义来自弗拉斯卡蒂手册 (the Frascati Manual, OECD, 2002)。该定义本来用以评估研 发活动, 但它已被包括学术机构在内的大部分社会群体广泛 采纳并应用。这本手册所说的 “研究” 是指 “为了增加人类、 文化和社会的知识储备而进行的系统性创造工作, 以及利用 这些知识储备进行新的应用的各种活动” (p30)。在英国的 学术研究质量评估中, 研究被定义为“调查引发新见解并且 能够有效共享的过程” (Higher Education Funding Council for England, 2011)。在这两个定义中有几个重要内涵。首先, 它 们都重点强调了知识可以是全新的, 也可以是如第二个定义 所指出的以某种方式运用已有的知识引导新的见解, 而这种 知识应当通过调查研究获得。调查研究的特征之一在于其系 统性, 这有别于一般的询问和其他形式的 “发现”世界的调 查。系统的调查研究是指缜密的数据收集、清晰的指导数据 采集和分析的概念性理论框架, 以及具备全面开展研究活动 的严谨性和技巧。其次, 这两个定义同时强调了研究即是一 个互动的过程 (信息不断被共享), 也是一个实践的过程 (强 调知识的 “应用”) 。

如上所述, 研究的第二个特征一一通过调查的过程获得 知识的应用一这对于规划学科尤为重要。很多规划研究工 作者都要求他们的研究工作在解决现实世界中的问题时有所 贡献, 有些人甚至希望通过他们的研究形成一个政策建议或 编制一个具体的规划。因此, 规划领域聚焦于规划实践。关 于这一焦点长期存在众多争论, 而且从未有可能形成统一的 答案。对于我们而言, 我们总是以城市未来发展为中心采取 集体的 “规划行动” (Healey, 2010; Albrechts, 2004)。这实际
上是一种城市治理的形式, 包含了将知识赋予规划行动可能 性的过程（Friedmann, 1987)。同时,正如最近多次被强调的, 知识不仅是先前提到的基于系统调查形式基础上研究产生的 知识, 即科学上证明可靠 (scientifically-robust) 的知识, 也 包括各类经验的、实用的和地方性知识 (Schön, 1983)。

那么系统调查在规划领域中扮演着何种角色呢? 本书的 编著者们提供了若干建议, 但是这些建议可能并不一致。概 括起来, 系统性研究调查起到对经验进行巩固和评估的作用 (是什么在起作用? 为了谁? 有什么利害敒关的条件一为 什么 (why) ? 什么时候（when）? 在哪儿（where）? 什么 程度 (how) ? 同时也起到对相关技术、工具、未来的可选 概念以及实践习惯的开发和评估的作用。很多规划研究同样 具有描述性和解释性, 直白一些, 也就是对 “到底是怎么回 事” 不断进行发问。在这方面, 规划研究更加靠近社会科学 和自然科学领域的研究调查传统。然而, 虽然规划研究与广 泛的社会科学或者自然科学研究的传统相呼应, 但它的一些 特征仍使得规划学科内的研究工作独具特色, 尽管这些特征 并非是规划学科所独有的。我们认为, 规划研究者进行系统 性调查、知识的生产和应用的这个独特环境, 是由这些特征 之间的组合、特征本身的显著性以及它们构建规划研究活动 的方式所造成的。我们认为以下内容是从事规划研究的关键 性学科特征, 其中很多特征都反映着与规划相关的更广泛的 问题 :

- 行动导向 ${ }^{1}$ : 要求在进行一个研究项目时关注展开该 研究活动的最开始的社会和政治动机, 以及该研究结果企图 给这些动机造成影响和变化的决心；

- 明确的规范性焦点 ${ }^{2}$ : 做规划研究涉及对价值的识别, 这些价值可以激发研究兴趣、并在某些案例中支撑着“想 要”在实践中产生实际影响的动机。规划研究往往在一个交 织着关于 “应该如何做” 的不同观点的复杂框架中进行。 因此, 研究作为一种产生知识和深刻见解的活动, 总会不可 避免地涉及到对权力关系的塑造;

- 认可系统性的知识产生在塑造和评价现实世界干预中 的价值;

- 对于场所特质和空间关系抱有实质的兴趣 ${ }^{3}$ ：认识到 许多不同的力量和关系塑造着场所特质的产生, 同时某一场 所发生的内容与他处发生的内容具有复杂的联系。从这个角 度上看, 规划研究与其他空间学科 (例如地理学) 具有一些 共同的特征。

(1) 参见弗里德曼和哈德森 (Friedmann \& Hudson, 1974) 关于规划中知识和行动关系的报告, 及弗里德曼 (Friedmann, 1987) 关于此报告发展的文献。

(2) 参见哈珀和施泰因 (Harper \& Stein, 1992) 为探索这个规范层面的特定规划的报告, 及豪 (Howe, 1990) 为进一步考虑规划的规范性道德的报告。

(3) 第二章希利 (Healey, 2010) 提供了一个关于规划此特性的入门报告, 并提供了一步阅读的参考文献。 
- 对于规律和范式多元性的敏锐程度 ${ }^{(1)}$ : 需要关注规划 领域中基于不同的研究传统下的认识论和本体论, 无论是在 直接的领域还是在交叉学科领域都一样;

- 对知识产生和运用的政治制度环境的认知, 需要意识 到谁有可能运用知识和知识可能怎样被运用 ;

- 对知识产生和运用的伦理维度的敏感性 ${ }^{(2)}$ : 涉及关注 明确是什么和是谁的价值观念存在其中, 以及规划研究者的 道德行为。

我们也认为, 规划研究者应当意识到并且理解所有这些 特殊性, 并且展现出其从事的规划研究设计与规划研究工作 的价值。规划学科中这些从事研究的特殊性使得之前介绍的 研究的正式定义可能发生一些偏差。例如, 从事系统性调查 往往意味着收缩议题以关注关键性的要素和关系, 这往往被 称为 “分析单位” (Yin, 2009; Remenyi, 2012)。这对于研究 从实践经验或目的中引发的热点议题的规划师来说可能是非 常困扰的。在行动模式中, 规划领域的工作者需要意识到他 们对特殊实际议题产生影响的多元维度和关系。一个关键性 的实际技能是将多元化的概念和要素 “集成” 和综合为一个 理念、规划或者策略, 以塑造、解释和证明特定的干预措施 ${ }^{3}$ 。 但是在研究模式中, 重点则转变为对可能仅有的一种显著关 系的深人探究。研究者面临的最大挑战在于制定出恰当的核 心调查关注点, 以及为了实现研究目的, 将什么问题置于脉 络背景当中。

许多规划研究的实用性和实践性特征及其行动导向同样 影响着规划研究行为。例如, 身处学术环境中的博士生和研 究者常常被认为是可以选择和塑造自己的研究课题以及调查 模式的 “自由角色”, 然而正如在其他各行各业中一样, 这 种情况鲜有发生 ${ }^{4}$ 。尤其是在规划领域, 系统性研究是在各 种各样的制度环境中, 伴随着赋予研究者的各类期待中展开 的。尽管研究者可能受到他们自身动机和研究调查激励的驱 使, 但仍然受限于某种方式中。博士生必须注意资助者的诉 求, 尤其是那些全部或者部分由合作或者赞助机构资助研究 的博士生, 同时还要注意他们导师的关注点。学者们被鼓励 从外部资源寻求和保证研究经费, 这就意味着资助者会相应 的产生特定的、并且少有协商余地的需求和期待。规划研究
者也可能在从事实际规划工作的机构和咨询公司工作, 这些 单位往往需要研究者专注于他们当下需求的研究。专业研究 机构和专业政策咨询机构也许会认可他们的工作人员从事系 统性研究活动的价值, 但它们也会有一些确定 “研究什么” 和 “如何获得结果” 的专门议程。在本书的最后一部分, 我 们探究了这些不同研究情况之间所产生的张力。然而, 所有 这些都需要谨慎的道德和社会政治敏感性。研究伦理学的课 程与文本往往关注研究者与研究 “受试者”之间建立联系的 恰当方式, 以及将证据与发现相关联的恰当方式。然而, 尤 其是在规划领域, 注意研究调查和资助者之间的关系伦理、 注意研究发现的使用者同样重要 ${ }^{(5)}$ 。规划研究者同样需要知 晓他们和其他研究者所从事研究的社会政治环境。研究可以 用于维持现状和资助者的既存利益, 也可以用于催发广泛的 授权变革。因此, 研究人员选择研究什么, 以及资助者将什 么确定为研究的优先项目是永远也无法做到中立的。

\section{3 规划学科的知识传统及其对研究实践的影响}

任何学科的研究实践都不可避免地受到更广泛的学术传 统和框架的塑造作用, 这使得每个学科具有特殊的形式和特 征。不同的知识范式与具体的研究设计和研究方法相一致。 因此, 一个学科 (例如规划学科) 吸收了优势知识传统的方 式将导致其与确定的研究方法具有密切关系。本节主要在于 提供一个针对规划思想发展方式的总结性介绍, 并解释这如 何导致某些特定的研究传统和方法被优待（或者说被强迫接 受)。对于那些首次接触规划学科的人而言, 应当作为关于 规划知识传统的关键部分进行阅读（Friedmann, 1987） (); 对有经验的规划研究人员来说, 只要能够认识主要轮廓内容, 并且领会到所涉及的不可避免简化了的内容就可以了。对于 所有读者而言, 我们希望能够建立多样规划知识传统与规划 研究设计及方法之间的关系。

\section{1 多重启发塑造下的规划制度和实践演变}

规划领域在逐步变为一个学术范畴之前, 是以大量实践 形式出现的, 到后来它才在相关学科的基础上建立起自己的 学术基础。这些实践具有与不同的国家相关的不同侧重点。

(1) 弗里德曼 (Friedmann, 1987) 有关规划中不同知识传统的经典报告帮助开启这一多样性探索。

(2) 参见罗 - 皮寇罗和托马斯 (Lo Piccolo \& Thomas, 2009) 关于规划研究伦理的论述, 亨德勒 (Hendler, 1995) 关于规划更广泛的道德的讨论。

(3) 参见戴夫欧蒂和彭德尔伯里 (Davoudi \& Pendlebury, 2010)、坎贝尔 (Campbell, 2012) 和基钦 (Kitchen, 2007) 第八章;

(4) 随着 “科学”成为一种调查方式, 从19 世纪的斗争发展出学术 “自由”的爱念。然而, 科学探究的大部分领域被实践推动着, 大学长期以来 一直致力于管理类的培训, 最初是为教会, 后来是为了国家。近日, “学术自由”的概念一直受到争议。缓慢科学运动对不断增长的特殊行业 和市场对研究和学术自由的丧失构成的压力提出了批评, 同时还提倡“公民的科学”。参见 slow-science.org。

(5) 参见罗・皮寇罗和托马斯 (Lo Piccolo \& Thomas, 2009)。

６）参见弗里德曼 (Friedmann, 1987)、希利尔和希利 (Hillier \& Healey, 2010)、阿尔门丁格 (Allmendinger, 2009) 及费恩斯蒂茵和坎贝尔（Fainstein \& Campbell, 2012)。 
不同政治动态和制度形式背景。19 世纪末的法国, 重点在 于城市设计和城市形态的 “现代化” 以塑造具有国际影响力 的传统。在德国和美国的一些地区, 对城市扩张的监管成为 关键问题, 以确保建设发展与基础设施的供给相协调。这便 产生了土地利用分区实践的广泛传播。在英国, 一些形式的 城市规划的动力最初来自 19 世纪工业化带来的日益拥挤和 不健康的城市中对于提升住房和基础设施条件的迫切需求, 控制城市蔓延进一步促进了小汽车的增加和伦敦铁路网络的 建设 ${ }^{(1)}$ 。2 0 世纪中叶, 许多国家开始关注区域景观管理, 并 雄心勃勃心地开始关注区域和国家的经济发展。而在完全的 社会主义国家中, 规划的焦点变成对整个经济体的规划 ${ }^{2}$ 。

这些不同的背景, 不仅对规划研究产生了多样的关注焦 点, 而且还要求规划师具备不同类型的专业知识。规划工作 和规划行业的整合, 在有些国家发源于建筑学, 在另一些国 家则发源于土地测量和工程学。在许多东欧国家, 规划工作 由接受过区域经济分析训练的人员主导。目前, 来自各种各 样学科背景的人们开始展露出规划的专门知识, 即便他们往 往并没有接受过正规的 “规划” 训练 ${ }^{3}$ 。

如此多元的实践渊源生成了适应于多元知识传统的规划 领域知识文化并不为奇。“规划理论” 作为巩固这些影响的 方式出现, 并且始终作为冲突性启发和传统的引导者 ${ }^{(}$。但 是这些讨论（或者是 “对话”）都围绕着本章开始时提出的 议题展开。然而, 理解这些议题的方式在不同的知识传统中 演化。在下面的小节中, 我们介绍这些知识传统及其对 “研 究活动” 的影响, 并且将它们与规划实践与制度环境联系起 来。在阅读以下内容时重要的一点在于铭记这些知识传统并 非以一个传统接替和覆盖较早的一个传统的形式或被安排在 一个历史性的连贯范式中, 其中很多传统持续并行存在, 导 致了学科多元化以及共存的知识传统的异质性。

\section{2 早期规划的发端 : 城市和区域规划中调查的演变}

在 19 世纪末和 20 世纪初早期的规划工作中, 并不关注 提升城市环境或者发展区域和国家经济, 规划研究的重点主 要在于 “寻找场所”。规划师需要空间布局和需求规模的信 息。这些现在在大多数西方国家能够取得的数据库当时尚未 产生。场所的特征、条件和潜力必须通过多种形式的 “调查”
获得 (5)。在这一 “调查”工作中, 三种规划传统可能被定义。 一是各种各样的数据收集一人口、住房、土地利用、不同 种类的就业等。这种类型的研究很少关注关于场所特质和动 态的假设, 而这些假设往往支持着考虑方面和数据收集的选 择。现在我们称这种研究为 “实证主义者”一一将对象定义 为简单的事实——和 “经验主义者”一一收集描述性的数据 而不仔细思考如何解释它的重要性。用这种方式来进行场所 研究被含蓄地视为现象的汇聚, 几乎不关注这些数据之间的 联系以及因果动态过程。二是受到 19 世纪法国地理学的影 响, 有着很强的综合性, 无论在区域还是城镇, 都强调场所 文化。受到这一传统影响的规划工作被鼓励考虑场所的 “精 神”。20世纪初伟大的规划先驱帕特里克 - 格迪斯 (Patrick Geddes）试图将这两种传统结合起来, 强调市民生活具有社 会性、经济性、政治性和生物性等多重方面, 强调实地观察 的重要性, 还有将这些知识带入场所的某种意义中, 以及 这些知识可能对未来的影响分析的必要性 (Geddes, 1915b, 1968)。它的工作被证明对很多后来抱有规划目标的城市和 区域规划研究具有启发性 ${ }^{\circledR}$ 。其中许多规划工作起初以咨询 的模式进行, 由创新专家来促进新的场所设计和管理的方法 论, 而非学术调查。一些规划顾问专家在新兴的规划教育项 目中进行教学, 这些教育项目是非常实际和实用的课程, 影 响了新一代的规划专家。

\section{3 社会科学转向 : 理性分析、系统建模和理论构建}

20 世纪 50 年代之前, 规划工作一直由建筑师、工程师 和土地测量师等这些与城市建设相关的人主导。到 20 世纪 50 年代以后, 社会科学开始被引入规划领域, 尽管基于历 史根源还可以追溯至 19 世纪中期（Friedman, 1987)。在社 会主义国家, 经济学家是设计和实施国家规划实践的关键专 家。在北美和欧洲, 经济学家创立了不同的规划原理和理论, 并且开始发挥对于公共政策设计的重要影响, 主要的方法源 自凯恩斯主义关于混合经济的论述, 其中民主国家提供框架, 在框架中资本运营被塑造并且被鼓励（Healey \& Hiller, 2008: introduction, P. xi)。规划的重点是保持物质增长和确保以公 平的方式进行利益分配。这对规划师来说, 需要具备比过去 的描述性和文化性规划研究更成熟的理解。正是在这一时期,

(1) 参见伯格和伦 (Berg \& Lune, 2012), 吉文 (Given, 2008) 和戈拉德 (Gorard, 2003), 作为例子可参见耶诺和施瓦茨谢伊 (Yanow \& SchwartzShea, 2006)。

(2) 参见萨克利夫 (Sutcliffe, 1981)、霍尔 (Hall, 1988)、沃德 (Ward, 2001) 及伯奇和西尔韦 (Silver \& Birch, 2009)。

(3) 参见罗德里格斯 - 巴奇列尔 (Rodriguez-Bachiller, 1991)。

(4) 参见规划理论杂志 (the journal of Planning Theory)、弗里德曼 (Friedmann, 1988)、戴夫欧蒂和彭德尔伯里 (Davoudi \& Pendlebury, 2010)。

(5) 参见格迪斯 (Geddes, ) 1915a/2000 和恩格斯 (Engels) 1845/2003，研究传统的早期例子。

（6) 参见霍尔 (Hall, 1996), 尤其是第五章; 梅勒 (Meller, 1990), 第六章关于格迪斯 (Geddes) 工作的影响; 沃德 (Ward, 2001)，关于 20世纪的 规划传统。 
在充满活跃辩论的美国早期规划院校, “规划理论”开始成 型 ${ }^{1}$ 。管理科学和制度经济学的发展也被用以发展成为规划 技术, 旨在帮助塑造城市与区域的发展战略和投资项目 ${ }^{2}$ 。 规划研究者被鼓励设计社会关系模型用以预测未来社会的情 景。这样的规划模型主要被用于特定的系统如运输动力学, 也有被用于整个经济系统和社会系统。随后爆炸性的研究可 以追溯到这一时期的规划学术期刊 ${ }^{3}$, 关注的焦点从描述性 研究（是什么? 在哪儿? 有多少? ) 转向事件及其结果产生 的关系研究、转向成因的研究一一即焦点转向 “怎么样” 和 “为什么” 问题的研究。

这对许多规划师来说, 这种社会科学的转向伴随着规划 工作的专业化培训扩大, 使规划领域在知识发展上向前迈进的 一大步, 并且影响了一代成长中的规划师。但是, 无论是历史 的还是从后来的时代来看, 规划领域的社会科学转向所体现出 的概念都受到质疑。后来, 将规划视为政治和经济背景的产物, 具有稳定的、线性的发展路径, 并将实现更加的经济社会繁荣 作为规划的目标, 这时的规划开始采用的概念假设知识模型主 要来自自然科学和新古典主义经济学模型, 期望规划实现是人 类和自然力量在一个地方的 “真正地” (即客观地) 相互作用 现象发生, 表现为“实证主义” 的规划假设, 规划研究的任务 在于产生关于生产关系的研究假设并且预测它们的发展过程, 寻找它们之间的因果关系和普遍规律, 为构建预测模型提供基 础。这些规划研究存在于很多研究中, 其中关于人类本性的假 设来源于新古典主义经济学。这些假设将社会视为由个体组成, 个体有其偏好和兴趣且能理性计算。这些假设被整合到一个 著名的规划过程模型 ${ }^{(}$, 模型传达的思想是 : 城市与区域总 体规划能够通过关系建模、偏好计算和结果预测的理性过程 编制出来, 同时嵌人技术发展要素, 对拟定的增长和发展路 径进行监测和评估。这些系统性技术、“理性”计算为包括规 划领域博士生在内的当时新兴的规划研究者提出了广泛的挑 战。但是到了 20 世纪 60 年代末, 这一规划研究设想同时受 到规划实践经验和保守的规划师的不断抨击。

\subsection{0 年代激进主义批判 : 科学规划、类方法和行 动规划研究兴起}

到了 1970 年代, 由于政府提升了对城市和区域发展的
干预程度, 对于具备规划技能的人才需求开始增加。这种转 向促进了大学和相关研究机构增加规划专业人才培养。在英 国等一些国家, 咨询活动逐渐萎缩, 并被各级政府和学术界 的规划师所取代。在有些地方, 学者承担了大量的咨询工作。 很多规划研究工作被大学、规划部门的研究团队和特殊的研 究机构承担。学术界已经成为规划领域的一支重要力量! 但 这时, 战后福特主义的增长动力已经趋缓, 一些先前的政策 限制开始显现, 将发展视为一个线性过程的理念逐渐显得缺 乏说服力, 政治体制稳定的假设也受到质疑, 增长的环境成 本变得越来越明显, 社会效益也未能满足最贫穷的社会阶层。 此外, 民权运动强调了社会分化不应仅仅归因于经济过程, 还与种族、民族、性别和其他形式的社会差异相关。这一时 期学校中很多规划学者对社会运动抱有同情, 这对已经建立 的规划方法论和规划实践造成了威胁。

这些鼓励采用更积极的方法进行研究。由于很多研究都 集中在不公平和效率低下的规划实践和政策措施的批评 ${ }^{(5)}$, 所以一些规划研究者展开了通过 “行动研究” 这类更加接近 激进主义实践的研究方法。尽管研究者常常受到不公平或者 已建立的实践环境的危险性的刺激, 但是他们仍渐进式探索 将这些感受植人到模型的更广泛的研究框架。对于许多人来 说, 马克思主义政治经济学的复兴正好提供了这样一个理论 框架。这对将社会视为一个集合进行理性计算的假设造成了 冲击。于是, 它取代了社会结构定位人的行为的社会模式, 并基于经济主导类别的 “结构性” 冲突构建了新的研究框 架。基于这样的理解: 如果冲突是社会过程固有的属性, 那 么将不会有政治和经济稳定性的假设存在 ; 资产阶级从工人 阶级获取剩余价值, 所以后者的贫困是由前者剥削式的增长 导向而导致的, 往往出于资产阶级的利益调节资本与劳动之 间的关系; 这样, 规划制度和规划实践就被被理解为是社会 调控（有时是剥削）的一部分。这种知识和政治取向导致了 规划研究的重点转向关注社会过程, 关注人类社会是怎样通 过社会过程被建构的? 关注城市治理是怎样形成的? 关注谁 从规划活动的结果中受益等问题 ${ }^{\circledR}$ 。这种社会结构和社会斗 争的动力学分析鼓励揭示资本主义剥削行为的研究, 这些说 明规划实践往往很难都是出于有益的目标而展开的。这也就 是说, 社会系统能被构建综合型模型并按照线性方式进行结

(1) 参见弗里德曼 (Friedmann, 1973)。

(2) 参见达尔和林德布洛姆 (Dahl \& Lindblom) 及西蒙 (Simon, 1945)。

(3) 这一时期的关键期刊是《美国规划师协会杂志》(JAIP: Journal of the American Institute of Planners), 随后是《美国规划学会杂志》(JAPA: Journal of the American Planning Association)。

(4) 参见泰勒 (Taylor) 1998 关于理性综合模型的解释和批评。

(5) 行为研究包含研究者的系统性反思; 参见里森和布拉德伯里 (Reason \& Bradbury, 2008) 和麦利夫和怀特海德 (McNiff \& Whitehead, 2006)、 Uttke 等人所著章节, 以及科特瓦尔和穆林所著本书第五章 (Kotval \& Mullin)。

(6) 费吕博基格 (Flyvbjerg, 2001) 认为“谁受益”是规划领域的关键性问题。 
果预测的有效性被阶级斗争的因素削弱了。如果模型预测的 结果是阶级斗争的结果, 那么线性预测显然不能有助于想象 末来。

20 世纪 70 年代发展出来的批判性评价工作提供了一个 实践价值的焦点一一做规划为了什么。人们开始聚焦实践, 聚焦规划工作包含些什么。一些研究者, 特别是那些不认同 政治经济学马克思主义模式的研究者, 提倡从关注设计抽象 决策技术转而关注规划实施的实践 ${ }^{1}$ 。另一些人开始注意到 规划实际上在做些什么 (2)。这便强调出机构的作用。人们可 能并不是理性的经济学计算师, 但是也并非仅仅作为广泛的 社会力量而存在。规划学者开始把目光转向运用其他哲学启 示来解释他们的发现, 并专注于他们的研究努力。

\section{5 后现代主义 / 后实证主义 / 后结构转向}

在 20 世纪 70 年代后半期以后, 尽管 “现代性” 和“现 代主义” 的一些要素在很多规划实践中仍然持续存在, 但其 概念范式 ${ }^{3}$ 却受到越来越多的挑战。在全球范围内, 传统的 物质持续增长 (“进步”) 的整体路径理念, 逐渐被不确定性 和不可预测性的认知所取代。社会民主的西方模式似乎无法 实现关于全体富裕的承诺, 而社会主义模式也遇到了腐败和 经济误判等政治问题。其他的社会也对唯物主义和西方模式 的殖民主义傲慢提出挑战, 并辅以学科内包括女权主义方法 在内的其他形式的知识批判。在此背景下 ${ }^{4}$, 规划的问题是 什么、为什么、如何处理城市发展问题受到了较之前而言更 多的争论。规划研究者开始转向本体论和认识论的思想确认 身份和知识, 认为到社会结构是复杂的, 而非如预定的 “现 实”那样简单。

这种哲学观念的转变在规划理论文献中得到大力促进和 讨论, 并表现为很多不同的方向 ${ }^{5}$ 。这些方向都受到 20 世 纪科学和社会科学提出的实证主义假设的批判。这一批判认 为, 由于人类情感的局限, 将永远无法把握人类所生活着的 物质的和 “真实的” 世界。我们了解的往往只是对所发生的 事件的一部分解释, 并且我们的解释无法避免不受到我们特 定的历史和地理条件的影响。正如舍恩（Schön, 1983）的名
言所说 : 这意味着人类, 尤其是专家学者们, 需要培养反思 其工作假设的能力。对于研究者而言更是如此。这一哲学观 点意味着批判性评价研究主要在于其是否揭示了隐藏在理所 当然的社会日常生活背后的社会过程, 而研究者接受这个观 点的同时也应当明确自己的假设。

在这一研究传统中, 出现了各种方式的分析政策 “话 语” 的爆炸性研究 ${ }^{6}$ 。有些研究探讨了利益相关者合作伙伴 关系的作用、参与和协同设计流程的运作 ${ }^{7}$ 。还有一些研究 关注规划者和规划过程的其他参与者实际上所做的内容及其 思考, 以及参与者对他们所处的情形进行分析 ${ }^{8}$ 。

这些工作的大部分都是由大学中的规划师和学者完成, 许多研究受到了政府和国际机构的资助。在方法论上, 这样 的研究广泛运用案例分析, 尤其与基于场所的空间学科如规 划相关。这些研究都是在结合多种研究方法, 包括文献分析, 参与者观察、调查和其他统计性的证据收集, 例如内容分析 法等研究策略基础上完成的。这种 “解释性” 研究面临的主 要挑战是其叙事方式的建构, 应当呈现出研究发现与概念和 问题（用以建构调查研究）之间的联系。这种解释性研究, 不同于从个别案例到群体案例的概括, 而是从概念框架中概 括主题 (Yin, 2009)。这个研究框架可能本身包含或甚至在 研究过程中不断发展。正像许多博士生的发现一样, 构建这 样的研究框架是一项很难做好的工作。但是, 这样的工作的 确极大地提升了我们对于规划实践的理解一一怎样规划和城 市发展进程是怎样工作的? 它们如何随环境而变化? 机构的 主动性和更广泛的力量之间是如何相互作用的? 这也突出了 规划领域规范性议程复杂性的重要程度。尽管规划研究的焦 点还在规划做什么、为什么做、谁受益, 但这些问题现在已 经转到必须重视身份和价值观的多样性和变动性等许多方面 的理解上。

这个 “解释性” 研究的传统已经被 “定性” 的社会科学 研究方法的文本扩展得到发扬光大 ${ }^{9}$ 。但是其哲学意义上的 转变可以被定位到更早期的系统分析的发展时期。而在 20 世纪 60 年代, 建立交通系统或土地利用系统模型的人们结 合系统趋于平衡态的系统动力学观点构想出一个具有稳定联

(1) 参见普莱斯曼和韦达夫斯基 (Pressman \& Wildavsky, 1973), 以及巴雷特和富奇（Barrett \& Fudge, 1981）。

(2) 参见福雷斯特 (Forester, 1989, 1999), 以及霍克 (Hoch, 1994)。

(3) 这一术语意味着用一个概念体系建构一个科学问题。

(4) 参见格里德 (Greed, 1994) 关于它们及其关系的论述。

(5) 参见希利和希利尔 (Healey \& Hillier, 2008) 的第三卷, 以及希利尔和希利 (Hillier \& Healey, 2010)。

（6) 参见哈耶尔 (Hajer, 1995) 环境相关论述和詹森和理查德森 (Jensen \& Richardson, 2004) 关于欧洲空间规划的论述。

（7) 参见希利（Healey, 1998, 2006)，以及英尼斯和布赫 (Innes \& Booher, 1999)。

(8) 参见图德一琼斯马克和托马斯休 (Tewdwr-Jones M \& Thomas H, 1998)。

(9) 一般方法参见西尔弗曼 (Silverman, 2011)、伯格和伦（Berg \& Lune, 2012)、耶诺和施瓦兹 - 谢伊 (Yanow \& Schwartz-Shea, 2006), 以及费吕博 基格 (Flyvbjerg, 2001) 和杰索普 (Jessop, 2005)。 
系的世界。但是, 新的分形几何学抛弃了这样的假设, 认为 世界和人们具有复杂的联系, 不确定性在复杂系统建模中 扮演者重要的角色, 应当成为规划的关键（Batty \& Longley, 1994; Openshaw \& Openshaw,1997; Batty, 2005; de Roo \& Silva, 2010)。同时, 规划领域开始充分理解冯诺依曼（von Neuman)、摩根斯坦 (Morgenstern)、乌拉姆 (Ulam)、图灵 (Turning) 的博亦论及微观一宏观行为观点。这项工作基 于这样的假设 : 自然过程不能有效地解释世界, 人类行为又 受到我们建构和作用于自然环境方式以及人类与自然相关联 方式的影响（同时也影响着后者）等。这一认知反过来要求 对于自然和人类系统（空间和空间特征）随着时间和空间进 化、适应过程的双重感知, 同时还要认识到不同的地方、行为、 模式和过程的创造性（Silva, 2011; Wu \& Silva, 2013, 2012)。

“一体万用” 模型的概念已经过时, 并转向寻求更加定制化 的途径, 着眼于方法论、模型、数据的组合以及所述一个或 其中几个最适合的问题和目标的选择的途径。在这个新的范 式下, 定制化需要具备向其他学科学习的开放理念, 并认识 到什么方法和数据是可用的, 认识到什么是可能的（什么在 当下是不可能的）以及摒弃我们已经习惯先人为主的确定性 的方法显得至关重要。

\section{4 规划研究的认识论和道德敏感性}

上述简要介绍了历史故事, 似乎好像是一个接着一个被 取代。现实上绝不是这样的, 所有传统的例子都能够在最新 出版的研究和出于特定实践目的的研究中找到。在可获取数 据和场所描述传统非常有限的国家, 基本的调研工作是必须 的。实证主义的假设存在于自然科学和经济学领域, 并渗人 与环境问题相关的规划领域, 并且在新的公共管理实践中不 断进化。在一些实践和研究中, “经验主义”仍然存在, 其 中因素和数据以非常分散的方式被使用。萨维茨基 (Sawicki, 2002）将 “指标” 运动描述为一种 “经验主义” 的神话, 为 实现政策目的，而很少具备系统性的概念架构。

此外, 前面提到社会理论和复杂性科学的发展有助于削 弱定量和定性研究方法论之间的传统区别。哲学的转变已经 削弱了认为运用科学能够完全理解世界的希望。相反, 大多 数研究者普遍认为, 任何研究都仅仅是认识复杂的、动态的、 我们生活着的、规划工作进行着的一部分世界。源于 “实证 主义”传统的方法仍然有效, 尤其是在研究背景可以被假设 是稳定的情况下, 其中就主要关系和含义达成合理性共识、 权力和不平等的存在并不能够妨碍其运用。“解释性”传统 下的工作可能有助于为统计方式的研究调查设定参数。
各种观点和研究传统的存在要求规划研究者解释其关注 的研究问题及进行研究设计时做出的选择。规划理论提供了 情景化和建构研究的方法。研究技巧在于以特定的方法提出 研究问题, 并且提供一些新的观点, 这些观点不仅仅能阐明 这个问题, 还能够引发重新概念化过程。此外, 在世界存在 不同的体制背景、权力关系和价值驱动理论下, 研究课题的 选择已经不再中立。根据不同的规划研究课题, 问题和成果, 规划研究既可以是开放的, 也可以是作为现存权力关系的一 种支撑。规划研究者需要意识到规划研究对于规划实践作用 的局限性。一方面, 实用性决策并不一定能从规划研究中 “读 取”, 规划研究的作用在于为规划工作的实用性判断提供支 持材料, 而并非取代实用性判断; 另一方面, 规划研究不应 与规划实践中的问题相脱离。然而, 在接触了最近后实证主 义哲学家和社会科学家的观点后, 一些博士生和学术论文作 者往往却沉迷于冗长的理论展示和叙述, 与随后实证调查的 联系非常微弱。总体而言, 21 世纪的规划研究者应避免简 单地提出异议, 而是谨慎地定位研究, 并向着富有想象的、 系统化的研究努力。

这些知识传统与规划学科总体轮廓交织在一起, 认为规 划研究者具有一系列的关键研究敏感性非常重要。这些最重 要的敏感性包括如下方面 :

- 对与其研究课题相关的社会背景的认识;

- 能够明确地阐明人或者其他力量可能做出怎样的行 为, 因此对于本体论的理解非常重要;

- 在有关 “知识是如何被产生和验证的假设中” 谨慎地 定位研究调查, 这需要对认识论有所理解;

- 针对适用于环境、问题和假设的研究策略选择, 能够 提出明确的理由 ;

- 仔细关注如何进行 “研究解释” 和 “研究报告” 的架 构和表达, 这包括根据听众和读者的反馈进行提升。

然而, 正如本文开头指出的, 规划领域研究的目的不仅 仅是为了取得知识的进步, 也是为了改革与评估, 甚至是为 帮助变革实践活动。

\section{5 在实践中研究: 研究与实践的相互作用}

理论和实践之间的关系常常是空间规划文献中被广泛讨 论的主题 ${ }^{1}$, 然而, 研究和实践之间的关系却较少被关注。 本文前面的部分定义了 “行动导向” 作为规划研究的特征, 尽管这仅仅是规划研究和实践领域之间存在的诸多关系之 一。规划研究和实践是多元的、复杂的关系, 其中一些关系 强调 “谁” 进行规划研究、利益相关者在研究过程中的作用、

(1) 参见亚历山大 (Alexander, 1997)、哈里斯 (Harris, 1997)、阿尔门丁格 (Allmendinger) 和图德 - 琼斯 (Tewdwr-Jones) 1997 关于关系交换的研究。 
从事和委托进行规划研究的人们之间的关系等重要问题。以 上反映出研究和实践之间的关系是很重要的。这里重点介绍 空间规划领域研究和实践之间的四个重要关系。测定和评论 这些关系的简单方法是它们是否给予 “研究者” 的立场以特 权, 今天这种做法正日益受到的挑战。

\section{1 实践是研究的 “思想” 源泉}

研究和实践的第一个重要关系在于, 实践为调查和研 究提供了问题的根源。这是一种知识的传统模式特征, 在 实践中, 实际问题会出现, 并受到包括大学在内的研究机 构的关注, 因此有用的知识得以产生, 并且反馈给实践者。 舍恩 (Schön) 在 1983 年明确记载了这一模式, 并且提出 了批判。这一模式的前提是明确区分学术机构中高水平研 究产生的知识以及舍恩提到的 “沼泽式低地” (Schön, 1983: 42-43)。事实上许多规划研究者寻求解决实践问题意味着实 践往往是系统研究调查思想的源泉。学习空间规划课程的 学生往往被要求定义一个实践中的问题作为他们调查研究 的焦点。这种 “实践的相关性” 在其他研究和实践的关系 方面也起着重要作用 (见前文), 但是此处强调将实践作为 疑惑、问题和研究调查地区的来源。一些组织, 如政府部 门和专业机构, 可以以重点议程的形式有效地设置研究优 先顺序, 以形成由其他人开展的研究。在其他情况下, 实 践中能够形成为研究焦点的 “问题” 本质可能非常不明显, 或者尚未被很好地定义, 因此研究者定义出 “问题” 就非 常重要。

\section{2 实践是开展学术研究的 “场所”}

实践常常是学术研究者开展实证研究的 “场所” 或 “地 点”, 其他感兴趣的人也可以在实践中 “做研究”。学术研究 者使用的 “田野” 这一术语是指去学术机构之外做实地调 查。田野通常包括实践的环境, 如规划办事处、公共空间、 受访者的工作场所等。在规划实践中所被称为的 “场所”, 意味着它是一个由学术机构单独独立出的 “地点”, 最终还 伴随着有一种或另一种已提取的实践数据。博士研究生在分 析数据前可能会用很长的时间去学校以外的地方进行实地调 研。这是一个理解研究过程的很传统的方式, 最近的研究成 果强调研究是一个集体活动, 鉴于研究开展的过程中活跃的 合作者, 研究活动涉及很多利益相关者 ${ }^{1}$ 。因此, “科学院” 和 “田野” 的传统区分受到了挑战并被重新评估, 这要求研 究者适应新的角色和开展研究的新机制。

\section{3 实践是从事研究的场所}

本文开头就强调了研究开展的制度和组织环境的宽广范 围, 其中很多便 “处于实践中”, 例如智库、政府部门和咨 询机构。研究任务被委托支持多重尺度上的政策准备和评估。 国家政府可能希望通过研究来支撑其规划政策, 地方政府也 可能希望研究为其规划的制定提供证据性的基础。研究和实 践之间的这一关系不同于学术研究, 它更强调研究作为实践 的一部分。因此, 做学术研究和在实践中做研究的相似之处 和不同之处便非常明确了。例如, 访谈作为一个学术研究项 目的一部分, 可能涉及录音、转录和编码等细节。而在实际 研究项目中, 只要注意几个关键要点作为备忘录即可。在这 样的环境中, 在文章开始的部分介绍了的系统性的研究活动 可能只能够支撑委托人短期和迫切的需求, 因为这种做法在 系统探究和学术研究的传统标准方面明显缺乏严谨性。“在 实践中进行研究” 的某些方面也可能被高度常规化、规范化 并建立起方法论和工具。因此, 很多影响学术研究特征性的 恰当的方法论的形成可能在实践中的体现并不明显。

\section{4 实践是学术研究的对象}

研究和实践之间的最终关系反映着研究者通过其研究活 动塑造实践的努力。这提出在一定程度上改变了早期定义的 研究以及通过研究所得到的学术交流成果 ${ }^{2}$ 。强调研究如何 影响实践, 重点越来越多的放在以证据为基础的政策制定和 研究的 “影响”, 这使得研究和实践处于另一种重要的关系 中一一研究的目的在于影响 “在实践中做什么”。在某些情 况下, 通过研究产生的见解已经对实践产生了明显的影响, 而困难的是使得研究能够长期、广泛地影响实践的内容。

\section{5 研究和实践的操控}

空间规划中存在一些有趣的方面, 即: 研究和实践的不 同关系将互动、结合产生一个特殊的研究环境。大学中从事 学术研究的研究者可能同样活跃在一些咨询项目中, 也可能 与私营部门的规划和研究咨询机构进行合作。因此研究者可 以跨越学术和专业背景积极参与专业网络, 这意味着他们可 能操控多个角色和机构, 有时接触规划实践, 有时参与实践 研究, 还可能是研究的用户, 这些在本书的第一部分传记性 的思考部分进行了很好的呈现。有时空间规划研究的专业化 背景还在开展研究的环境中加人了另一个重要的维度, 其中 部分学术研究人员和规划院校形成更广泛的专业和多学科项 目的一部分。对于任何从事规划研究的人而言, 最重要的是

(1) 参见马克思一尼夫 (Max-Neef, 2005)、卡西纳里和莫拉尔特 (Cassinari \& Moulaert) 5.3 节。

(2) 参见戴夫欧蒂 (Davoudi) 5.2 节, 以及戴夫欧蒂 (Davoudi, 2006) 和克里泽 (Krizek et al., 2009)。 
识别和反思他们与实践相关的 “立场”, 并理解这一立场对 其所从事的研究的影响。

\section{6 结论 : 对着手规划研究者的善意提醒}

我们以一系列有用的提醒来作为本书导论的总结。首先, 规划研究是一个系统性的探究过程, 然而我们也在本文中将 研究行为描述为 “技巧”。规划领域研究与探究是具有吸引 力的、创造性的工作, 这在本书第一部分的个人思考中有所 体现。规划研究需要我们将观察得到的理解与关键性的、富 有想象力的深刻见解相结合。这需要具备结合分析和解释技 能开展系统性探究的能力。规划领域研究调查 “技巧” 涉及 许多复杂的判断, 这将影响到研究如何发起、如何聚焦、如 何开展及如何撰写。这些判断都不仅与知识取向和分析的一 致性相关（虽然它们很重要）, 还受到制度环境和从事特定 研究的原因的影响。因此研究者应当在研究的早期阶段停下 来思考其研究在怎样的制度和环境中发起和开展, 出于什么 目的、研究最终试图呈现怎样的成果。

其次, 规划的研究工作, 尤其是相比于其他自然科学 和社会科学, 往往具有强烈的实用目的导向。因此, 从事 规划研究的人们往往遇到 “那又怎样? ” 这一问题的挑战。 你应该在别人问你这个问题之前问问自己，并通过思考准 备自己的答案。这种强烈的实用导向也要求同时尊重研究 设计和撰写研究成果, 尊重研究伦理。这些伦理道德的挑 战涉及尊重性的关心和研究者预计如何对待研究对象等问 题。在这样的挑战中, 研究人员有望依据道德守则进行处 理 ${ }^{(1)}$ 。在规划领域, 规划研究者还需要具备对于实践和所处 体制环境的特殊性和对我们的研究发现可能被运用的方式 的敏感性。这包括对于研究选题的社会政治意义的敏感性, 以探讨你的研究活动和实践之间的关系, 并仔细思考研究 的伦理问题。

第三, 要记住规划是空间性的学科, 大部分研究的重点 在于探究、评估并试图理解场所及其相互关系。研究往往专 注于发现和解释特定场所是如何工作的。同时还应从对比研 究中学习其他地方的规划经验 (Sanyal, 2005)。规划研究者 应当在规划研究中保持对特性的聚焦以形成规划研究基础, 并尝试解释不同的地方不同的结果。

最后, 《规划研究方法手册》(The Routledge Handbook of Planning Research Methods) 将呈现出有经验的规划研究者如 何发现并制定出路径, 以迎接做研究中复杂方法的挑战。然 而, 任何一位编著者都不会提供一种精准的关于如何做研究 的 “秘诀”, 即使这是可能实现的。一个标准的“秘诀” 可
能会扭曲引领规划研究者探究特定的情形及问题的方向。最 终每个规划研究者要自己制定、证明并解释自己的判断, 专 注于将自己关心的议题与合适的概念视角连接起来, 以架构 一个研究。定义关于这个议题有什么可研究的, 并将此转化 为一个研究问题、一个研究策略、一系列合适的研究方法和 分析、报告所发现内容的方式。我们希望这本书中的材料将 有助于呈现、启发和充实这些判断。鉴于不同的哲学方法论 塑造形成的本体论和认识论共同影响着规划领域, 今天规划 领域的研究者必然在冲突的立场中把握研究路径, 并将特殊 问题概念化、设计出适当的研究策略。因此, 研究者应该思 考自己在研究过程中如何操控路径, 随着研究进展不断调整 方向, 并仔细思考选择某一路径而非其他路径的理由。 UP I

\section{参考文献}

[1] ALBRECHTS L. Strategic (Spatial) planning re-examined[J]. Environment and Planning B: Planning and Design, 2004, 31: 743-758.

[2] ALEXANDER E R. A mile or a millimetre? measuring the 'planning theory-practice gap' [J]. Environment and Planning B: Planning and Design, 1997, 24(1): 3-6.

[3] ALLMENDINGER P. Planning theory[M]. 2nd edition. London: Palgrave Macmillan, 2009.

[4] ALLMENDINGER P, TEWDWR-JONES M. Mind the gap: planning theorypractice and the translation of knowledge intoaction; a comment on Alexander[]]. Environment and Planning B: Planning and Design, 1997, 24: 802-806.

[5] BARRETT S, FUDGE C. Policy and action[M]. London: Methuen, 1981.

[6] BATTY M. Cities and complexity[M]. Cambridge: MIT Press, 2005.

[7] BATTY M, LONGLEY P. Fractal cities: geometry of form and function[M]. New York: Academic Press, 1994.

[8] BERG B, LUNE H. Qualitative research methods forthe social sciences[M]. Cambridge: Pearson, 2012.

[9] BIRCH E L, SILVER C. One hundred years of cityplanning's enduring and evolving connections[J]. Journal of theAmerican Planning Association, 2009, 75(2): 113-122.

[10] CAMPBELL H. Planning to change the world: between knowledge and action lies synthesis[J]. Journal of PlanningEducation and Research, 2012, 32(2): 135-146.

[11] CLAMP C G L, GOUGH S, LAND L. Resources for nursing research[M]. 4th ed. London: Sage, 2004.

[12] DAHL R A, LINDBLOM C E. Politics, economicand welfare[M]. 4th edition. New Brunswick: Transaction,1953/1992.

[13] DAVOUDI S. Evidence-based policy: rhetoric and reality[J]. The Planning Review, 2006, 42, 165: 14-24.

[14] DAVOUDI S, PENDLEBURY J. The evolution of planning asan academic discipline[J]. Town Planning Review, 2010, 81(6): 613-645.

[15] DE ROO G, SILVA E. A planner's encounter withcomplexity[M]. Aldershot: Ashgate, 2010.

[16] Economic and Social Research Council. ESRC Framework for Research Ethics. Swindon: ESRC, 2012.

[17] ENGELS F. The great towns[M] // LEGATES R, STOUT F, eds. The City Reader. 3rd ed. London: Routledge, 1845/2003: 58-66.

[18] FAINSTEIN S S, CAMPBELL S, eds. Readings in planning theory[M]. Oxford: Wiley-Blackwell, 2012

(1) 这些准则因学科的不同而异。英国一个适用于社会科学研究的框架来自经济与社会研究委员会 (ESRC, UK)。 
[19] FLOWERDEW R, MARTIN D. Methods in human geography: a guide for students doing a research project[M]. 2nd ed. Harlow: Pearson, 2005.

[20] FLYVBJERG B. Making social science matter: why socialinquiry fails and how it can succeed again[M]. Cambridge: Cambridge University Press, 2001.

[21] FLYVBJERG B, LANDMAN T, et al, eds. Real social science: applied phronesis[M]. Cambridge: Cambridge University Press, 2012.

[22] FORESTER J. Planning in the face of power[M]. Berkeley: University of California Press, 1989.

[23] FORESTER J. The deliberative practitioner: encouraging participatory planning processes[M]. London: MIT Press, 1999.

[24] FRIEDMANN J. Re-tracking America: a theory of transactive planning[M]. New York: Anchor Press, 1973.

[25] FRIEDMANN J. Planning in the public domain[M]. Princeton: Princeton University Press, 1987.

[26] FRIEDMANN J. Planning theory revisited[J]. European Planning Studies, 1987, 6(3): 245-253.

[27] FRIEDMANN J, HUDSON B. Knowledge and action: a guide to planning theory[J]. Journal of the American Institute of Planners, 1974, 40(1): 2-16.

[28] GEDDES P. City survey for town planning purposes, of municipalities and government[M]// LEGATES R, STOUT F eds. The City Reader. London: Routledge, 1915a/2000: 330-335.

[29] GEDDES P. Cities in evolution[M]. London: Ernest Benn, 1915b/1968.

[30] GIVEN L, ed. The sage encyclopaedia of qualitative research methods[M]. London: SAGE, 2008.

[31] GORARD S. Quantitative methods in social science: the role of numbers made easy[M]. London: Continuum, 2003.

[32] GREED C. Women and planning: creating genderedrealities[M]. London: Routledge, 1994.

[33] HAJER M. The politics of environmental discourse[M]. Oxford: Oxford University Press, 1995.

[34] HALL P. Cities of tomorrow[M]. Oxford: Blackwell, 1988.

[35] HALL P. Cities of tomorrow. an intellectual history of planning and design in the twentieth century[M]. Updated ed. Oxford, UK: Blackwell, 1996.

[36] HARPER T L, STEIN S M. The centrality of normative ethical theory to contemporary planning[J]. Journal of Planning Education and Research, 1992, 11(2): 105-116.

[37] HARRIS N. Orienting oneself to practice: a comment on Alexander[J]. Environment and Planning B: Planning and Design, 1997, 24(6): 799-801.

[38] HEALEY P. Collaborative planning in a stakeholder society[J]. Town Planning Review, 1998, 69(1): 1-21.

[39] HEALEY P. Collaborative planning: shaping places infragmented societies[M]. 2nd edition. Basingstoke: Pal-graveMacmillan, 2006.

[40] HEALEY P. Urban complexity and spatial strategies: towards a relational planning for our times[M]. London: Routledge, 2007.

[41] HEALEY P. Making better places: the planning project inthe twenty-first century[M]. Basingstoke, UK: Palgrave Macmillan, 2010.

[42] HEALEY P, HILliER J. Foundations of the planning enterprise[M] // Critical Essays in Planning Theory, 1. Aldershot: Ashgate, 2008.

[43] HENDLER S, ed. Planning ethics: a reader in planning theory, practice and education[M]. New Brunswick, NJ: Rutgers, 1995.

[44] Higher Education Funding Council for England. Research Excellence Framework 2014. Assessment Framework and Guidance on Submissions. Bristol: HEFCE, 2011.

[45] HILLIER J, HEALEY P, eds. The Ashgate research companion to planning theory[M]. Aldershot: Ashgate, 2010.

[46] HOCH C. What planners do: politics, power and persuasion[M]. Chicago: Planners Press, 1994.

[47] HOWE E. Normative ethics in planning[J]. Journal of Planning Literature, 1990, 5(2): 123-150.

[48] INNES J E, BOOHER D. Consensus building as role playing and bricolage: toward a theory of collaborative planning[J]. Journal of the American Planning Association, 1999, 65(1): 9-26.
[49] JENSEN O B, RICHARDSON T. Making European space: mobility, power and territorial identity[M]. London: Routledge. 2004.

[50] JESSOP B. Critical realism and the strategic-relational approach[J]. New Formations, 2005, 56(1): 40-53.

[51] KITCHEN T. Skills for planning practice[M]. Basingstoke: Palgrave, 2007.

[52] KRIZEK K, FORSYTH A, SLOTTERBACK C S. Is there a role for evidence-based practice in urban planning and policy?[J]. Planning Theory and Practice, 2009, 10(4): 459-478.

[53] LEWIS-BECK M S, BRYMAN A, LIAO T F. The sage encylopaedia of social science research methods[M]. London: SAGE, 2004.

[54] LOGIO K A, DOWDALL G W, BABBIE E R, et al. Adventures in criminal justice research[M]. 4th ed. London: Sage, 2008.

[55] LO Piccolo F, THOMAS H, eds. Ethics and planning research[M]. Aldershot: Ashgate, 2009.

[56] LYONS E, COYLE A. Analysing qualitative data in psychology[M]. London: Sage, 2008.

[57] MAX-NEEF M A. Foundations of transdisciplinarity[J]. Ecological Economics, 2005, 53(1): 5-16.

[58] MCNIFF J, WHITEHEAD J. All you need to know about action research[M]. London: SAGE, 2006.

[59] MELLER H, GEDDES Patrick. Social evolutionist and city planner[M] London: Routledge, 1990.

[60] OPENSHAW S, OPENSHAW C. Artificial intelligence in geography[M]. Chichester: John Wiley, 1997.

[61] Organisation for Economic Co-operation and Development. The Measurement of Scientific and Technological Activities: Proposed Standard Practice for Surveys on Research andExperimental Development. Frascati Manual. Paris: OECD, 2002.

[62] OUTHWAITE W S, TURNER S, eds. The sage handbook of social science methodology[M]. London: SAGE, 2007.

[63] PRESSMAN J L, WILDAVSKY A B. Implementation: how great expectations in washington are dashed in Oakland[M]. Berkeley: University of California Press, 1973.

[64] REASON P, BRADBURY H, eds. The sage handbook of action research: participative inquiry and practice[M]. London: SAGE, 2008.

[65] REMENYI D. Case study research. The quick guide series[C]. Reading: Academic Conferences, 2012.

[66] RODRIGUEZ-BACHILlER A. British town planning education: accomparative perspective[J]. Town Planning Review, 1991, 62: 43-445.

[67] ROY A. City requiem, calcutta: gender and the politics of poverty[M]. Minneapolis: University of Minnesota Press, 2003.

[68] RUANE J M. Essentials of research methods: a guide tosocial science research[M]. Malden, MA: Blackwell, 2004.

[69] SANYAL B. Comparative planning cultures[M]. Abingdon, UK: Routledge, 2005.

[70] SAWICKI D. Improving community indicators: injecting moresocial science into a folk movement[J]. Planning Theory and Practice, 3(1), 2002: 13-32.

[71] SCHÖN D. The reflective practitioner[M]. New York: BasicBooks, 1983.

[72] SILVA E. Cellular automata models and agent base modelsfor urban studies: from pixels, to cells, to hexa-dpi's[M]// YANG Xiaoyun ed. Urban remote sensing: monitoring, synthesis and modeling in the urban environment. Hoboken, NJ: Wiley-Blackwell, 2011: 323-345.

[73] SILVERMAN D, ed. Qualitative research[M]. London: SAGE, 2011.

[74] SIMON H. Administrative behavior[M]. New York: Free Press, 1945.

[75] SOMEKH B, LEWIN C. Theory and methods in social research[M]. 2nd edition. London: SAGE, 2011.

[76] SUTCLIFFE A. Towards the planned city: Germany, Britain, the United States and France, 1780-1914[M]. Oxford: Blackwell, 1981.

[77] TAYLOR N. Urban planning theory since 1945[M]. London: Sage, 1998.

[78] THYER B. The handbook of social work research methods[M]. London: Sage, 2010.

(下转 142 页) 